\title{
Application of Computer-Aided Translation Technology in Translation Teaching
}

\author{
http://dx.doi.org/10.3991/ijet.v8i5.2926 \\ Zhang Erwen, Zhang Wenming \\ Anhui Polytechnic University, Wuhu, China
}

\begin{abstract}
-this paper aims to advocate the application of CAT technology in the traditional translation teaching class in universities. Based on the introduction of the development of CAT technology and its wide application in modern translation industry, the necessity of applying CAT technology in translation teaching is convincingly proven, which is followed by an elaboration on the design of CAT teaching modules and the construction of CAT teaching system. At the end of this paper, the practice of enhancing the traditional translation curriculum with reference to application of CAT technology is clearly demonstrated so as to set an empirical example to those colleges and universities who'd like to follow suits.
\end{abstract}

Index Terms-Computer-Aided Translation, Translation Memory, Translation Teaching, Curriculum Enhancement.

\section{INTRODUCTION}

21 st century is an era of information explosion and global integration. Along with the development of economy, science and culture and the booming of international exchange, the demand on translation in various fields is increasingly swollen, which calls for more and more translators competent in translating large quantity of materials in various applied fields. However, the traditional translation class, as teacher-centered, is oriented in teaching of translation theories and techniques, but neglects the practicability of translation course and the ultimate goal of cultivating students' translation ability. Therefore, it becomes a crucial issue to reform the traditional translation class so as to meet the development of society.

Since 1980s, with the popularity of multimedia computers and the emergence of global network, computeraided translation (abbreviated as CAT hereafter) technology has aroused great interest among researchers, and the huge potential of CAT teaching in translation class has been recognized by many language teachers. It is a must to apply the fruit of advance in science to traditional translation class and establish a CAT teaching mode in modern information age.

This paper, by introducing the development of CAT technology and such concerning concepts as MT (Machine Translation) and TM (Translation Memory), advocates the application of CAT technology in translation teaching and, by proposing a practicable mode of CAT teaching, holds that, students majoring in translation, only after being able to use different CAT software, can claim to be a translator competent enough to meet the requirements of the market.

\section{REVIEW OF CAT TECHNOLOGY}

\section{A. From MT to CAT}

MT, abbreviation of Machine Translation, also referred to as Automated Translation, is "a sub-field of computational linguistics that investigates the use of computer software to translate text or speech from one natural language to another."[1] Research on MT started as early as in 1933 when the Russian scientist P. P. Telojamsky proposed a detailed step for using machine in translation. In 1954, the first MT system invented by Georgetown University and IBM successfully translated a Russian material of about 250 words into English, which marked the birth of MT system. In 1976, Canadian Bureau of Translation developed TAUM-METEO translation system to translate weather report, which was the milestone in the history of MT and marked the applicability of MT technology.

However, since the birth of MT technology, the accuracy of MT has been widely questioned by researchers. The readability of translated text and the coverage of MT system on linguistic phenomena are far from satisfactory. Therefore, researchers began to resort to the development of CAT technology.

CAT, abbreviation of Computer-Aided Translation, is "the process whereby human translators use computerized tools to help them with translation-related tasks."[2] It is a translation strategy that translators use computer program to handle part of the translation process.[3] CAT is different from MT mainly in that humans are pivotal to the process of translation. A CAT tool is meant to support a human translator in his/her work to speed up the translation and provide consistent terminology while machine translation is meant to stand alone as much as possible. In CAT, the computer program supports the translators, who translate the text themselves. In MT, the computer program translates the text, with no human intervention during the translation process. [4] "The hope was to combine the best of both paradigms: CAT, in which the human translator ensures high-quality output, and MT, in which the machine ensures a significant gain in productivity.[5]

\section{B. The core of CAT: TM}

TM, abbreviation of Translation Memory, is a kind of mechanism which can store and repeatedly use texts that have been translated. The concept of TM originated in the 1970 s, but only since the late 1990s has this type of tool developed into a significant commercial entity. At first, the scheme of translation memory mechanism was proposed by Peter Arthern, who pointed out that the translator can gain a lot if he can search the stored similar texts online. "The pre-requisite for implementing my proposal 
is that the text-processing system should have a large enough central memory store...the organization in question should store all the texts it produces in the system's memory."[6] "...previous translations are stored in the computer and retrieved as a function of their similarity to the current text being translated."[7] From what was discussed above, it is shown that TM is a type of linguistic database that is used to store source texts and their translations, which are broken down into short segments that often correspond to sentences.

The operation principle of TM is that users can create one or more translation memory databases by using the stored source text and translated text, and during translation process, the system will automatically search the stored translation resources for the same or similar translation, so that the user can focus on the translation of the new content instead of doing meaningless repetitive work.[8] "The translation unit saved in the translation memory consists of a source language segment and its target language equivalent."[9] When a translator has a new segment to translate, the TM system consults the database to check whether this new segment corresponds to a previously translated segment and presents the translator with the previous translation as long as a matching one is found. The translator can thus review the previous translation and decide whether or not to incorporate it into the new translation.

TM is the core of CAT technology. The computer searches and maintains the databases in the background while the translator translates with the help of the software. When translating, the system automatically searches for the most similar existing translation units in the database(s) and prompts to the user. Therefore, the translator can take advantage of the stored translation units instead of translating the same content twice. He can also edit and revise the automatically translated texts in the target language, which greatly improves the translation efficiency and guarantees the consistence of the translation of terminology.

\section{CAT Tools}

Along with the development of TM, emerge variety of CAT tools such as TRADOS, SDLX, Déjà Vu, MemoQ, OmagaT, Star Transit and IBM Translation Manager, which feature personalized interface, supporting multiple file formats, powerful terminology management and simple automatic search function. These CAT tools, on one hand, witness the popularity of CAT technology in the process of translation, and on the other hand, represent the new standard in training qualified professional translators. In another word, nowadays, it is indispensible for professional translators to master such CAT tools in their practice of translation.

\section{NECESSITY OF APPLYING CAT TECHNOLOGY IN TRANSLATION TEACHING}

It is widely acknowledged that the purpose of educating students is to meet the demand of society. In this information explosion era, when CAT becomes a dominant technology in translation industry, it is of great necessity for educators to acquaint the students majoring in translation with CAT technology, which will be demonstrated as follows:

\section{A. Trend for the development of translation as a discipline}

CAT technology represents the trend for the development of translation as a discipline in this information age. As was discussed above, nowadays it has become an indispensable ability for translators to utilize CAT tools so as to produce high-quality, high-efficiency translations in limited time. Therefore, in order to cultivate students majoring in translation who can catch up with the development of society, educators in the university must take actions in applying CAT teaching in translation course. Only when the students majoring in translation acquire CAT technology at school can they meet the new requirement of the future society on translation and produce satisfactory translations after graduation.

\section{B. Advantage in job-hunting}

It becomes a big advantage for translation students in job-hunting if they've acquired CAT technology. Nowadays, most translation companies require that their employees, whether full-time or part-time, should master the skill of translating with the aid of CAT software such as TRADOS and Déjà $\mathrm{Vu}$, etc. and receive and submit their work by internet. If students majoring in translation don't learn to utilize those main CAT tools at school, it will be extremely hard for them to be adapted to the working procedure of translation companies and face the fierce competition in the future job-hunting market after graduation.

\section{Powerfulness in terminology management}

The traditional translation course still focuses on themes of literature and humanities. However, according to statistics, in the present translation market, the nonliterary translation amounts to $95 \%$ of the total output,[10] which covers fields such as insurance, telecommunication, law, costume, engineering, aeronautics, chemical industry, metallurgy, environmental protection, finance, economy and energy, etc. In these fields, there are a lot of fixed terminology and expressions, which requires terminology management of CAT tools to standardize the work of translators.

\section{Activeness in interaction}

The traditional translation class, as teacher-centered, focuses on exploration of translation theories and techniques, but ignores the practicability of the translation course and the ultimate goal of cultivating and improving students' translating ability. By adopting CAT teaching system, a student-centered, computer-and-internet-based translation teaching platform can be founded to establish and continuously enrich the resource base of translation teaching, and to carry out active interactions between teacher and students and among students, which will not only improve the teaching effects, but also stimulate students' positivity in learning translation.

\section{E. Cultivation of comprehensive ability}

The traditional translation class succeeds in teaching students various translation theories and techniques, but fails in cultivating students' comprehensive ability of applying what they've learnt in class to analyze and solve concrete problems in their practice of translation. CAT teaching system, based on huge translation resources, discussion between teacher and students and online exchange among students, can profoundly ameliorate the situation 
by improving students' ability in language switch, text comparison and information acquisition.[11]

\section{DESIGN OF CAT TEACHING MODULES}

Although CAT has acquired eye-catching achievement in recent years in China, it hasn't received corresponding development in translation teaching in universities. So far there are only ten universities establishing CAT course, i.e. Peking University, Beijing International Studies University, Beijing University of Aeronautics \& Astronautics, Sun Yat-sen University, Beijing Foreign Studies University, Shandong Normal University, Hebei Normal University, the Chinese University of Hong Kong, City University of Hong Kong and University of Macau, among which, Shandong Normal University is the earliest university carrying out CAT teaching practice among their undergraduates and postgraduates majoring in translation. Based on an empirical study on the CAT teaching practice in the above-mentioned ten universities, a practical CAT teaching module is designed to form a comparatively comprehensive teaching content system, which can be divided into the following four modules.

\section{A. Translation information technology}

The emergence of CAT system is treated as the most significant technology renovation in translation industry, which is highly valued by translation researchers. Monterey Institute of International Studies (MIIS) proposed that "computer is an essential tool for translators."[12] Therefore, the first CAT teaching module concerns translation information technology. A fluent mastery of digital word processing technology is the pre-requisite for students majoring in translation to use CAT system, the teaching of which consists of the following five sub-modules.

Advanced word processing skills: Modern word processing software is no more treated as a typewriter transplanted to the computer. However, according to a survey conducted by IT industry in $2009,80 \%$ of the computer users only know $20 \%$ of the functions in Word, which means there are a lot of unknown advanced techniques "hidden" in the word processing system. And it is these techniques that help improve the working efficiency of translation. The word processing techniques learners of CAT technology in translation class must acquire mainly include advanced searching \& replacing, automatic generation of catalog \& index, cross reference, macro, revision of marked documents, automatic sequence and so on.[13]

Digital text accessing skills: Using CAT system requires having the electronic document in the source language before the process of translation. However, a great number of documents to be translated are presented in hard copy to translators in reality. In order to convert the hard copy into the electronic form, Optical Character Recognition (OCR) Technique is needed. Students majoring in translation are required to learn the differences of various OCR software, their applied occasions and how to get the optimal recognition effect, etc. Besides, they are supposed to be taught how to get the reliable electronic documents by web P2P or contacting the publisher or the author.

Input technology: Although English has no so-called "input method", Chinese does have choices as to input them. Nowadays, the whole sentence input technology has been developed so maturely that it can greatly improve the work efficiency of translation. In addition, advanced voice recognition system is available for translators who are competent in sight translation. How to evaluate different methods of input and take advantage of them in the practice of translation is also a technique students majoring in translation need to acquire in class.

Searching technology: All kinds of searching engines on the internet and academic databases make logic operations according to Rules and Laws of Boolean Algebra. To learn the advanced searching techniques would help solve problems that searching only by keywords fail to. This part also includes the introduction of electronic dictionaries and encyclopedia.

Corpus searching: This sub-module focuses on corpus technology, which is of prime importance to the application of CAT technology in translation. It's been proven that with reference to the corpus acquired by searching on the internet, the quality of translation from Chinese to English can be highly improved.[14] Wang Kefei holds that bilingual parallel corpus has wide application prospect and potential exploitation value in translation teaching, and using parallel corpus is convenient for finding translations of special expressions, which, by providing alternative translations, makes the translation of terminology more accurate and idiomatic than that given by bilingual dictionaries.[15] Students majoring in translation will be taught the concept, construction and searching techniques of bilingual corpus, and the significance and ways of fully applying corpus searching in the practice of translation will also be discussed.

\section{B. Terminology management}

It is acknowledged by many CAT software users that terminology management is the most important task in the practice of translation, whose value even surpasses that of TM because in the process of translation, the repetition rate of terminology is far beyond that of complete translation unit. In order not to search the specific terms every time they begin a new translation, translators should use a terminology management system (TMS), which can not only help with various aspects of terminology-related tasks, including the storage, retrieval and updating of term records, but also ensure greater consistency in the use of terminology so as to make the translation easier to understand and prevent miscommunication.

In translation software, there are some specialized terminology tools, such as MultiTerm, Lexicon and Terminology in Déjà Vu X, etc. This kind of software has some basic functions of corpus analysis, which can rank the word frequency of source text, helping translators locate terminologies from the perspective of word frequency. Concerning terminology assortment, TRADOSER can be applied to automatically recognize and arrange mixed English and Chinese words, which can convert irregular word list into one with a tab in-between, making it much easier to import terms searched from the internet into a CAT system.

\section{Application of CAT system}

This module is the core of the whole course. As computer has become the primary tool for translators, CAT technology is the core of techniques they should master. In this module, some main CAT software will be introduced to the students, such as Déjà Vu X, SDL TRADOS, Wordfast and Google Translator Toolkit, etc., which are 
chosen from the perspective of the acknowledgement from users, the occupancy of market, the overall development of the software and the advancement of technology. The above-mentioned software stands for CAT systems of three interface types: word-processing plug-in interface (Wordfast), independent translation table interface (Déjà Vu X, SDL TRADOS 2009) and intelligent contrast interface (Google Translator Toolkit). In classroom teaching, a particular demonstration will be conducted on the systems of above-mentioned CAT software which combine translation project management, translation memory, translation interface and quality control. Concerning students' practice, it will be conducted in the form of workshop.

\section{Translation project management}

Any translation curriculum, if market-oriented, should be designed to take translation as an integrated industry process rather than a language switch process from the source text to the target one. Therefore, terms in management science such as project analysis, project management and quality control should be brought into the teaching content of translation curriculum. Only those who realize the importance of project management and quality control and acquire corresponding abilities can be better adapted to modern translation industry process. As the last step in translation project management, quality control plays a vital role in ensuring the translation quality. CAT software can offer a multi-dimensional resolution for quality control, which shall also be conducted in the classroom.

\section{CONSTRUCTION OF CAT TEACHING SYSTEM}

What was discussed above answers two questions: Why we should teach CAT technology in translation class and what to teach? However, it is far more difficult to construct a teaching system than to design the teaching content. Due to the shortage of qualified teachers and related hardware and software facilities, even some key universities in China find it difficult to carry out CAT teaching in translation class.[16] In order to guarantee the application of CAT technology in translation class and its effect, a comprehensive CAT teaching system shall be constructed, which is demonstrated as follows:

\section{A. Reform in teaching concept}

In the past several decades, great changes have taken place in every aspect of our society, which, as manifested in translation, is the conversion from traditional translation process to an informationalized and commercialized translation mode. Nowadays, most of translation projects feature large quantity, limited time and high demand on quality, which can not be handled by the traditional translation mode and call for modern means of project management and translation technology. CAT technology is designed from the very beginning to assist translators in accelerating translation speed and reducing repetitive work, which meets the demand of modern market and shows powerful vigor. At present, CAT technology has been adopted by most large-scaled translation service suppliers and multinational corporations, who declare that it's a pre-requisite for staff engaged in translation industry to acquire such skills. Therefore, to catch up with the development of society, translators are required to master not only the bilingual knowledge and cultures, but also the relevant advanced technologies.
As the major force in cultivating future employees engaged in translation industry, the administrators and teachers of corresponding majors in colleges and universities must be well aware of the above-mentioned conversion and take actions in adjusting and renovating the curriculum and adding some new courses related to translation technology, so as to make the teaching concept in accordance with the social development and industrial demand. In this way, compared with the students cultivated under the traditional curriculum system, those who finish the new courses can show greater priority in knowledge structure and practical skills and have more choices in the future employment. However, to achieve this, it requires the great vision in teaching concept and the joint efforts of administrators and teachers.

\section{B. Cultivation of teachers' practical ability}

As a newly emerging technology, CAT technology, although widely adopted in modern translation industry, is still an unfamiliar field to most translation teachers and remains unfrequented in translation teaching,[17] which brings to us a harsh question: how could students competent in using CAT technology in their translation be cultivated by inexperienced or even incompetent teachers? Therefore, to carry out CAT teaching in translation class requires that teachers acquire the relevant practical ability in operating CAT technology. Universities should attach much importance to the pre-service and in-service training for teachers, especially training on computer-based multimedia and network technology, and invite some experts in the application of CAT technology to supervise the real classroom teaching of those inexperienced teachers.

\section{Utilization of internet resources}

With the fast development, the internet has already become a database of abundant resources, from which both the teachers and students can get benefit. Such websites as Wikipedia, Baidu Encyclopedia, CNKI and some online databases and dictionaries can provide the beginners with a lot of basic knowledge about CAT technology. For example, an input of "computer-aided translation" on Wikipedia will be instantly responded with relevant information about CAT, MT and CAT tools. Therefore, in order to fully utilize the rich resources from the internet, CAT teaching should be conducted in classrooms with access to the internet, and students should be repetitively advised to utilize the internet as much as possible.

\section{Renovation in teaching methodology}

Personalization and differentiation in teaching: CAT teaching, compared with other linguistic education, requires much more on students' ability of using computer, which, due to the fact that students might come from different regions, for example, the rural area or the urban area, presents a diversified picture. Therefore, it is of great importance for teachers to make a comprehensive investigation on students' background and their current levels of using computer before they decide the teaching methods, the teaching courseware and the teaching content. In the teaching process, teachers should adopt varied teaching methods according to students of different characteristics. After class, they are encouraged to use BBS forum, QQ chat room and blog, etc. to answer questions from students so as to enhance the communication between teachers and students and achieve a real sense of individualized instruc- 
tion. Only in this way can personalization and differentiation be realized to maximize the effect of CAT teaching.

Cooperation among students: CAT technology is a totally new subject to all the students taking this course. Therefore, it's really a tough task for them to understand and master the relevant knowledge and practice on their own in a limited time. Under this circumstance, the teamwork and cooperation among students are advocated to help learn from each other so as to make greater progress at a faster pace.

Choice of corpus: To better demonstrate the advantages of CAT technology in translation, it is suggested to choose the corpus comparatively normative in both content and form, for example, science literature, financial report and political literature, etc. These corpus feature limited vocabulary, high repetitiveness of words, simple sentence pattern and accurate expression, which are very convincing in elaborating the functions of database, terminology management, alignment and TM.

\section{E. Communication with other teaching institutes}

As was discussed above, so far there are only ten universities in China which carry out CAT teaching in translation class, which means CAT teaching in universities still belong to a pilot study. Therefore, it would be wise for any university who starts to bring CAT technology into translation teaching to communicate with those ten universities, from which their good experiences can be learnt and the mistakes they made can be avoided.

\section{F. Contacts with CAT software suppliers}

CAT technology has been widely applied in translation market and thus gained a promising prospect in translation industry, which drives the main CAT software suppliers to continuously update their products so as to occupy a larger market share. Therefore, contacts with CAT software suppliers would definitely make CAT teaching in translation class more in accordance with the development of CAT technology, by which students can get access to the latest software and be more competent in their future practice of translation.

\section{ENHANCEMENT OF TRANSLATION CURRICULUM}

Even with the successful construction of CAT teaching system, it is still an unprecedented practice for colleges and universities to apply CAT technology in translation curriculum, which may bring about a lot of problem during the practice. After three years' practice of applying CAT technology in translation teaching in our university, some good experiences regarding the enhancement of translation curriculum have been accumulated, i.e. distribution of teaching contents and their time allotments, which will be demonstrated as follows and set as an example for those colleges and universities who'd like to follow suits.

\section{A. History of MT and CAT}

Knowledge on the history of MT and CAT is of vital importance to students, which can help them eliminate their misunderstanding on CAT. For many years, most people have mistaken CAT for MT and thought CAT were fully automatic. Considering the unsatisfactory results produced by current automatic translation systems, they hold great doubts on learning CAT technology or even reject it. Therefore, teaching on the history of MT and CAT could enable students to have a rational and objective expectation on CAT.

In our practice, we mainly focus on the introduction of the history and status quo of CAT to students, which takes 4 teaching hours.

\section{B. Principles of CAT}

Principles of CAT have mainly undergone two periods from rule-based basis, which includes the levels of word, sentence and semantics, to corpus-based basis. Acquaintance of such knowledge will definitely enable students to better understand the working mechanism of CAT tools.

In our practice, considering our students are art students, we prefer to illustrate these principles by citing simple examples and some charts, so that students can have a general understanding on them. For this part, we take 6 teaching hours.

\section{Translating tools in broad and narrow sense}

Translation tools in broad sense refer to all the software and hardware facilities which provide convenience during the translation, including word processing software and equipment, scanner, taping equipment, text formatconverting software, electronic dictionary and online dictionary; while translation tools in narrow sense refer to the CAT software designed specifically for the improvement of translation efficiency and optimization of translation process.

In our practice, we mainly focus on the translation tools in narrow sense, i.e. CAT and its relevant knowledge and products, whose main functions, such as terminology base, matching, TM, project management and quality guarantee, are introduced to students, which take 6 teaching hours.

\section{Corpus and CAT}

Corpus plays a vital role in the ultimate quality of translation, whose definition, classification, construction and maintenance will be discussed in the class.

In our practice, students are taught to use one or several corpus analysis tools, such as AntConc, MonoConc, ParaConc and WordSmith. For this part, we take 6 teaching hours.

\section{E. Terminology and terminology base}

Consistency in terminology also helps guarantee the quality of translation. For a large translation project, the advantage of having a terminology base is self-evident.

In our practice, the definition, classification and usage of terminology are taught firstly, which is followed by the introduction of the constructing, editing, enlarging and maintaining of terminology base. Some main terminology base tools, such as AnyLexic: Terminology Management 2.0, Sun Gloss and T-Manager 4.5, are also introduced to students. For this part, we take 10 teaching hours.

With all the above-mentioned contents introduced into translation curriculum, students reported they felt more confident in handling the translation of various materials. And the final examination, which took place after the total 32 hours' teaching, also revealed students' competence and performance in translation were highly improved. 


\section{CONCLUSION}

With the booming demand of society on translation, the traditional translation and teaching mode can no longer satisfy the modern translation industry. As CAT technology has made great progress in development and been widely applied in translation industry, it hasn't received the due attention in the traditional class of translation teaching. This paper, by a clear demonstration of the necessity of applying CAT teaching in translation class and a detailed manifestation of designing CAT teaching and constructing CAT teaching system, which are followed by the practice of enhancing the translation curriculum in our university, calls for the immediate action taken in the reform of the traditional translation teaching mode. Only by applying CAT technology in translation teaching, can translation education in universities catch up with the development of this information age.

\section{REFERENCES}

[1] A. Alcina, "Translation Technologies: Scope, Tools and Resources", Target, vol. 20, no. 6, pp.79-102, 2008. http://dx.doi.org/10.1075/target.20.1.05alc

[2] L. Bowker, Computer-aided Translation Technology: A Practical Introduction, Ottawa: University of Ottawa Press, 2002.

[3] J. C. Sager, Language Engineering and Translation: Consequences of Automation. Amsterdam \& Philadelphia: John Benjamins, 1995.

[4] M. Lebert, "Computer-Assisted Translation (CAT): Glossary", In Proceedings of Net des etudes francaises-Dossiers du NEF, pp. 2130, 2011.

[5] S. Barrachina, et al., "Statistical Approaches to Computer-Assisted Translation", Computational Linguistics, vol. 35, no. 11, pp. 3-28, 2009. http://dx.doi.org/10.1162/coli.2008.07-055-R2-06-29

[6] P. Arthern, Machine Translation and Computerized Terminology Systems: A Translator's Viewpoint, Amsterdam: North-Holland, 1978.

[7] H. Somers, The Translator's Workstation Computer and Translation: A Translator's Guide, Amsterdam \& Philadelphia: John Benjamins, 2003b
[8] Yanfu Fu, "Translation Memory Theory and Several CAT Software", Journal of Hunan Medical University, vol. 12, no. 25, pp. 22-27, 2010.

[9] C. Shih, "Translation Memory: A Teaching Challenge", Perspectives: Studies in Translatology, vol. 13, pp. 205-214, 2005. http://dx.doi.org/10.1080/09076760508668992

[10] Changshuang Li, Non-literary Translation Theories and Practice, Beijing: China Translation \& Publishing Corporation, 2004.

[11] Xiaofeng Zhong, "CAT Technology and Translation Teaching", Education and Profession, vol. 33, no. 15, pp. 125-126, 2010.

[12] Jun Wen, Et al., “A Study on MTI Curriculum Setting”, Foreign Language Teaching, vol. 30, no. 13, pp. 92-95, 2009.

[13] Bin Xu, "CAT: Teaching and Course Offering", Shanghai Journal of Translators, vol. 4, no. 10, pp. 45-49, 2010. http://dx.doi.org/10.1007/s11741-010-0109-3

[14] Xiuwen Cen, Et al., "Teaching Methodology of Internet and Workshop-based Non-literary text Translation", Shanghai Journal of Translators, vol. 1, no. 7, pp. 58-61, 2008.

[15] Kefei Wang, “Application of Bilingual Parallel Corpus in Translation Teaching", CAFLE: Computer-Aided Foreign Language Education, vol. 6, no. 14, pp. 27-32, 2004.

[16] Gangqiang He, "Tradition, Characteristics and Teachers-My View on the Construction of Translation Major", Shanghai Journal of Translators, vol. 3, no. 20, pp. 49-51, 2007.

[17] Bin Xu, Current Situation and Problems of CAT Training in Mainland China, Hongkong: the Chinese University of Hongkong, 2009.

\section{AUTHORS}

Zhang Erwen is with Anhui Polytechnic University, Wuhu 241000 China (e-mail: zhangerwen81@163.com).

Zhang Wenming is with Anhui Polytechnic University, Wuhu 241000 China (e-mail: 13956183660@ 163.com).

Manuscript received 19 June 2013. Published as re-submitted by the authors 13 October 2013. This work was supported in part by key project of Humanities and Social Sciences, Anhui Educational Commission under Grant 2011sk204zd and the teaching and research project of Anhui Polytechnic University under Grant 2010xjy35. 